\title{
Single-cell mass cytometry of microglia in major depressive disorder reveals a non-inflammatory phenotype with increased homeostatic marker expression
}

\author{
Chotima Böttcher [1]', Camila Fernández-Zapata @i]', Gijsje J. L. Snijders², Stephan Schlickeiser, \\ Marjolein A. M. Sneeboer ${ }^{2,4}$, Desiree Kunkel ${ }^{5}$, Lot D. De Witte ${ }^{2,4,6}$ and Josef Priller ${ }^{1,7,8}$
}

\begin{abstract}
Stress-induced disturbances of brain homeostasis and neuroinflammation have been implicated in the pathophysiology of mood disorders. In major depressive disorder (MDD), elevated levels of proinflammatory cytokines and chemokines can be found in peripheral blood, but very little is known about the changes that occur directly in the brain. Microglia are the primary immune effector cells of the central nervous system and exquisitely sensitive to changes in the brain microenvironment. Here, we performed the first single-cell analysis of microglia from four different post-mortem brain regions (frontal lobe, temporal lobe, thalamus, and subventricular zone) of medicated individuals with MDD compared to controls. We found no evidence for the induction of inflammation-associated molecules, such as CD11 b, CD45, CCL2, IL-1 $\beta$, IL-6, TNF, MIP-1 $\beta$ (CCL4), IL-10, and even decreased expression of HLA-DR and CD68 in microglia from MDD cases. In contrast, we detected increased levels of the homeostatic proteins P2Y $_{12}$ receptor, TMEM119 and CCR5 (CD195) in microglia from all brain regions of individuals with MDD. We also identified enrichment of non-inflammatory CD206 ${ }^{\text {hi }}$ macrophages in the brains of MDD cases. In sum, our results suggest enhanced homeostatic functions of microglia in MDD.
\end{abstract}

\section{Introduction}

Major depressive disorder (MDD) is one of the most common mental disorders across the lifespan and represents a leading cause of disability worldwide ${ }^{1}$. MDD is more prevalent in women than men and it increases the risk of suicide, obesity, and coronary heart disease ${ }^{2}$. Recent genome-wide association studies have associated MDD with variants in genes involved in hypothalamic-pituitary-

\footnotetext{
Correspondence: Chotima Böttcher (chotima.boettcher@charite.de) or Josef Priller (josef.priller@charite.de)

'Department of Neuropsychiatry and Laboratory of Molecular Psychiatry, Charité - Universitätsmedizin Berlin, Berlin, Germany

2Department of Psychiatry, Brain Center Rudolf Magnus, University Medical Center Utrecht, Utrecht, The Netherlands

Full list of author information is available at the end of the article These authors contributed equally: Chotima Böttcher, Camila FernándezZapata, Lot D. De Witte, Josef Priller
}

adrenal (HPA) axis function, neuronal differentiation, synaptic transmission, cytokine production, and immune response $^{3}$. Cell type-specific methylome studies have confirmed the involvement of the innate immune system in $\mathrm{MDD}^{4}$. The polygenic risk for MDD is moderated by environmental factors, such as childhood trauma ${ }^{5}$. Strong gene-environment interactions exist between HPA axis genes (Corticotrophin releasing hormone receptor 1, FK506 binding protein 5) and $\mathrm{MDD}^{6}$. Notably, higher FKBP5 expression promotes nuclear factor (NF)- $\mathrm{kB}$-mediated peripheral inflammation and chemotaxis ${ }^{7}$. Along these lines, chronic stress and low-grade inflammation are believed to contribute to the pathophysiology of $\mathrm{MDD}^{8-10}$. In fact, patients with MDD express increased levels of proinflammatory cytokines like interleukin (IL)-1, IL-6 and tumor necrosis factor (TNF)- $\alpha$ in peripheral blood,

\section{(c) The Author(s) 2020}

(c) (i) Open Access This article is licensed under a Creative Commons Attribution 4.0 International License, which permits use, sharing, adaptation, distribution and reproduction cc) in any medium or format, as long as you give appropriate credit to the original author(s) and the source, provide a link to the Creative Commons license, and indicate if changes were made. The images or other third party material in this article are included in the article's Creative Commons license, unless indicated otherwise in a credit line to the material. If material is not included in the article's Creative Commons license and your intended use is not permitted by statutory regulation or exceeds the permitted use, you will need to obtain permission directly from the copyright holder. To view a copy of this license, visit http://creativecommons.org/licenses/by/4.0/. 
which can access the central nervous system (CNS) and activate tissue-resident macrophages like microglia, impair HPA axis function, modulate monoaminergic neurotransmission, and reduce neural plasticity ${ }^{11-13}$. The tryptophan-kynurenine pathway links depression and inflammation, and under stressful and inflammatory conditions, microglial indoleamine 2,3-dioxygenase (IDO) activity reduces serotonin availability and results in the production of excitotoxic metabolites such as quinolinic $\operatorname{acid}^{8,9}$. In recent years, peripheral blood cytokines have been proposed as biomarkers of $\mathrm{MDD}^{14}$, and combinations of pro- and anti-inflammatory cytokines (e.g. IL-10) can help predict the response to antidepressant treatment ${ }^{15-18}$.

However, it remains an unresolved question of high therapeutic relevance whether the inflammation in MDD originates primarily in the periphery or in the CNS. Peripheral blood monocytes of MDD patients express higher levels of inflammatory/immune mediators like IL-1 $\beta$ and IL-6, as well as TNF, TLR2, CEBPA, and CCL2 mRNAs $^{19-24}$. In contrast, studies of CNS microglia have resulted in contradictory findings. Microglia are specialized tissue macrophages that originate from the yolk sac during early embryonic life, and play essential roles in brain development and maintenance of CNS homeostasis $^{25}$. Neuropathological examination of post-mortem frontal lobe tissue from MDD cases revealed increased numbers of activated microglia expressing ionized calcium-binding adaptor molecule (Iba) $1^{26}$ or quinolinic $\operatorname{acid}^{27}$. The number of primed Iba1-positive microglia and CD45-immunoreactive perivascular macrophages was increased in depressed suicides ${ }^{28}$. In contrast, many other studies did not detect significant changes in the density of major histocompatibility complex HLA-DRimmunoreactive microglia in frontal lobe, temporal lobe, thalamus or brain stem of MDD $\operatorname{cases}^{29-32}$. Gene expression profiling of post-mortem frontal lobe tissue from psychotropic drug-free persons with a history of MDD revealed increased expression of IL1A, IL3, IL5, IL8, $I L 10$, but not $I L 6$ or $T N F^{33}$. Gene expression of $T N F$, IFNG, and CCL2 was even reduced in the prefrontal cortex of depressed suicides ${ }^{28}$. Strong support for the neuroinflammation hypothesis of MDD comes from positron emission tomography (PET) studies of translocator protein $18 \mathrm{kDa}$ (TSPO) binding in depressed individuals with MDD. TSPO binding was found to be elevated in frontal lobe, temporal lobe and thalamus of depressed patients, and correlated with the severity of depression $^{34}$, cognitive dysfunction ${ }^{35}$, and the duration of antidepressant treatment ${ }^{36}$. However, no correlation with peripheral inflammatory markers like IL-1 $\beta$, IL-6, TNF, and C-reactive protein (CRP) was detected ${ }^{34}$. It is also still controversial whether TSPO binding is a good indicator of microglial activation in the human brain ${ }^{37}$.
Given that the role of microglia is not yet clear in major depression $^{38}$, we decided to use single-cell high-dimensional mass cytometry (CyTOF) to examine microglia from different post-mortem brain regions of medicated individuals with MDD compared to controls. We have recently demonstrated that this technique allows us to detect subtle phenotypic differences of human microglia across different brain regions with good correlation between post-mortem tissue and fresh brain biopsies ${ }^{39}$. In this study, we determined 59 protein markers at the single-cell level that unequivocally distinguish microglia from other brain macrophages and peripherally derived immune cells. The results suggest a non-inflammatory phenotype of microglia with increased homeostatic marker expression in MDD.

\section{Methods}

\section{Human post-mortem tissue}

Human post-mortem brain tissue was obtained from the Psychiatric Donor Program of the Netherlands Brain Bank (NBB-Psy; www.brainbank.nl). The Netherlands Brain Bank received permission to perform autopsies and to use tissue and medical records from the Ethical Committee of the VU University Medical Center. Tissue was collected post-mortem from donors from whom full consent had been obtained during life to conduct brain autopsy and research. Mediacted MDD cases $(n=6)$ were defined as donors with a diagnosis of MDD according to the DSM-IV or III. Control donors $(n=5)$ were defined as donors without a history of depression, confirmed by retrospective medical chart review. Detailed donor information is provided in Supplementary Tables 1-3.

\section{Microglia isolation}

Microglia were isolated from post-mortem brain tissue as described previously ${ }^{39}$. After autopsy, tissue was stored in Hibernate medium (Invitrogen, Carlsbad, CA, USA) at $4{ }^{\circ} \mathrm{C}$ until further processing. Microglia isolation started as soon as possible, at the latest after $24 \mathrm{~h}$. A single-cell suspension was generated by mechanical and enzymatic digestion with collagenase (3700 units/mL; Worthington, USA) and DNase $(200 \mu \mathrm{g} / \mathrm{mL}$; Roche, Switzerland) for frontal lobe (GFM), temporal lobe (GTS) and thalamic (THA) tissues, or $0.2 \%$ trypsin and $30 \mathrm{mg}$ DNase for subventricular zone (SVZ) tissue. A Percoll (Amersham, Merck, Germany) gradient was generated to separate viable cells from myelin, cellular debris, and erythrocytes. The middle layer was collected and washed twice, followed by positive selection of myeloid cells with CD11bconjugated magnetic beads (Miltenyi Biotec, Germany) according to the manufacturer's protocol. MACS-isolated $\mathrm{CD}_{11 \mathrm{~b}^{+}}$cells were fixed with fixation/stabilization buffer (SmartTube) and frozen at $-80^{\circ} \mathrm{C}$ until analysis by mass cytometry $^{39}$. 


\section{Intracellular barcoding for mass cytometry}

MACS-isolated CD11b ${ }^{+}$cells were thawed and subsequently stained with premade combinations of six different palladium isotopes: ${ }^{102} \mathrm{Pd},{ }^{104} \mathrm{Pd},{ }^{105} \mathrm{Pd},{ }^{106} \mathrm{Pd}$, ${ }^{108} \mathrm{Pd}$, and ${ }^{110} \mathrm{Pd}$ (Cell-ID 20-plex Pd Barcoding Kit, Fluidigm). This multiplexing kit applies a 6-choose-3 barcoding scheme that results in 20 different combinations of three Pd isotopes. After 30 min staining at room temperature, individual samples were washed twice with cell staining buffer $(0.5 \%$ bovine serum albumin in PBS containing $2 \mathrm{mM}$ EDTA). All samples were pooled together, washed, and stained with antibodies.

\section{Antibodies}

Anti-human antibodies (Supplementary Tables 4 and 5) were purchased either pre-conjugated to metal isotopes (Fluidigm), or from commercial suppliers in purified form and then conjugated by us using the MaxPar X8 kit (Fluidigm) according to the manufacturer's protocol. Each antibody was titrated and validated using different cell types from different body compartments, as described previously ${ }^{39}$.

\section{Cell surface and intracellular staining}

After cell barcoding, washing and pelleting, the combined samples were stained and processed as described previously $^{39}$. Briefly, cells were resuspended in $100 \mu \mathrm{l}$ of antibody cocktail directed against cell surface markers (Supplementary Tables 4 and 5) and incubated at $4{ }^{\circ} \mathrm{C}$ for $30 \mathrm{~min}$. Then, the cells were washed twice with cell staining buffer (PBS containing $0.5 \%$ bovine serum albumin and 2mM EDTA). For intracellular staining, the stained (non-stimulated) cells were incubated in fixation/ permeabilization buffer (Fix/Perm Buffer, eBioscience) at $4{ }^{\circ} \mathrm{C}$ for $60 \mathrm{~min}$. After two washes with permeabilization buffer (eBioscience), the samples were stained with antibody cocktails directed against intracellular molecules (Supplementary Tables 4 and 5) in permeabilization buffer at $4{ }^{\circ} \mathrm{C}$ for $1 \mathrm{~h}$. Cells were subsequently washed twice with permeabilization buffer and incubated overnight in $4 \%$ methanol-free formaldehyde solution. The fixed cells were washed and resuspended in $1 \mathrm{ml}$ iridium intercalator solution (Fluidigm) at room temperature for $1 \mathrm{~h}$, followed by two washes with cell staining buffer and two washes with $\mathrm{dd}_{2} \mathrm{O}$ (Fluidigm). Finally, cells were pelleted and kept at $4{ }^{\circ} \mathrm{C}$ until CyTOF measurement.

\section{Bead staining}

For the bead-based compensation of the signal spillover, AbC total antibody compensation beads (Thermo Fisher Scientific) were stained with each of the antibodies used in all three antibody panels according to the manufacturer's instructions. Stained beads were then measured with CyTOF and the compensation matrix was generated as described previously ${ }^{40}$.

\section{CyTOF measurements}

Cells were analyzed using a CyTOF2 upgraded to Helios specifications, with software version $6.7 .1014^{39}$. The instrument was tuned according to the manufacturer's instructions with tuning solution (Fluidigm), and measurement of EQ four element calibration beads (Fluidigm) containing ${ }^{140 / 142} \mathrm{Ce},{ }^{151 / 153} \mathrm{Eu},{ }^{165} \mathrm{Ho}$, and ${ }^{175 / 176} \mathrm{Lu}$ served as a quality control for sensitivity and recovery. Immediately prior to analysis, cells were resuspended in $\mathrm{ddH}_{2} \mathrm{O}$, filtered through a $20 \mu \mathrm{m}$ cell strainer (Celltrix, Sysmex), counted and adjusted to $3-5 \times 10^{5}$ cells $/ \mathrm{ml}$. EQ four element calibration beads were added at a final concentration of $1: 10 \mathrm{v} / \mathrm{v}$ in order to normalize the data to compensate for signal drift and day-to-day changes in instrument sensitivity. Samples were acquired with a flow rate of 300-400 events/s. The lower convolution threshold was set to 400, with noise reduction mode turned on and cell definition parameters set at event duration of $10-150$ pushes (push $=13 \mu \mathrm{s}$ ). The resulting flow cytometry standard (FCS) files were normalized and randomized using the CyTOF software's internal FCSProcessing module on the non-randomized ("original") data. The default settings in the software were used with time interval normalization ( $100 \mathrm{~s} /$ minimum of 50 beads) and passport version 2. Intervals with less than 50 beads per $100 \mathrm{~s}$ were excluded from the resulting FCS file.

\section{Mass cytometry data processing and analysis}

Following the workflow from our previous study ${ }^{40}$, Cytobank (www.cytobank.org) was used for initial manual gating on live single cells and Boolean gating for debarcoding. Nucleated single intact cells were manually gated according to the signals of DNA intercalators ${ }^{191} \mathrm{Ir} /{ }^{193} \mathrm{Ir}$ and event length. For de-barcoding, Boolean gating was used to deconvolute individual samples according to the barcode combination. Prior to data analysis, each FCS file was compensated for signal spillover using $\mathrm{R}$ package CATALYST ${ }^{41}$. For dimensionality reduction, visualization and further exploration, (2D)tSNE maps were generated based on the expression levels of all markers in each panel. For embedding, we set hyperparameters to perplexity of 30 , theta of 0.5 , and iterations of 1000 per 100,000 analyzed cells. To visualize marker expression, arcsinh transformation was applied to the data. All FCS files were then loaded into $\mathrm{R}$ and further data analysis was performed with a custom written script based on the workflow proposed by Nowicka and colleages $^{42}$. Briefly, for unsupervised cell population identification, we performed cell clustering with the FlowSOM $^{43}$ and ConsensusClusterPlus ${ }^{44}$ packages using all markers in each panel. We then performed visual inspection of cluster-colored tSNE plots and phenotypic heat maps for a more detailed profile of each cluster, and we determined the number of meta-clusters with consistent phenotypes 
for statistical testing. Based on visual inspection of t-SNE plots and heat maps generated at the merging step, a final number of meta-clusters was chosen that merged clusters into populations with consistent phenotypes (with a minimal mean frequency of $0.1 \%$ of parent $)^{40}$.

\section{Statistical analysis}

No randomization and blinding strategies were applied in this study. However, data processing and analysis, as well as statistical testing were carried out in an unsupervised manner. Dichotomous variables of the sample cohort were analyzed with Fisher's exact test (GraphPad Prism). Quantitative data are shown as independent data points with Box-Whisker. Exploratory analyses of statistical significance were performed using multiple $t$-test available through GraphPad Prism 8 with a false discovery rate (FDR) adjustment at $10 \%$ using the Benjamini-Krieger-Yekutieli procedure for multiple hypothesis testing, unless otherwise stated. A $P$ value $<$ 0.05 was considered statistically significant.

\section{Results}

\section{Samples}

MDD and controls did not differ in age, gender or postmortem characteristics (Supplementary Table 1). The average post-mortem delay was $8 \mathrm{~h}$ (range $4 \frac{1}{2}-12^{3} / 4 \mathrm{~h}$ ). Both groups were medicated, and donors differed with regard to medication used and psychiatric history (Supplementary Tables 2 and 3).

\section{Regional diversity of human microglia is preserved in MDD}

Human microglia (huMG) were isolated from postmortem brain tissue of subventricular zone (SVZ), thalamus (THA), temporal lobe (GTS) and frontal lobe (GFM) (Fig. 1a). In order to minimize the run-to-run variation and to facilitate the comparison of cellular profiles from different brain regions and individuals, we simultaneously profiled huMG samples from different brain regions of donors with MDD and controls in the same run. To do so, huMG were intracellularly barcoded using different combinations of palladium isotopes as described previously $^{39}$. Up to twenty samples were pooled, split equally and stained with two different antibody panels (Supplementary Tables 4 and 5). The antibody Panel A (36 antibodies) was designed to distinguish the major circulating immune cell subsets, including T \& B lymphocytes, monocytes, natural killer (NK) cells, from CNS immune cells, including microglia and border-associated macrophages. The panel also focused on cytokines, chemokines and other inflammatory mediators, and comprised antibodies against $\mathrm{P}_{2} \mathrm{Y}_{12}$ receptor, TREM2, CD45, CD3, CD14, CD16, CD11c, CD64, CD11b, CD56, EMR1, CD115, CD47, CD19, HLA-DR, CD56, CD68, CD33 (Siglec-3), CD192 (CCR2), CD195 (CCR5), CX3CR1,
CD141, CD32, CD206, CD163, MIP-1 $\beta$, IL-10, CCL2, IRF8, TGF $\beta$, and TNF (Supplementary Table 4). The antibody Panel $B$ was designed to investigate functional and activity changes in huMG subsets using 35 antibodies, including HLA-DR, IKZF1, ALDH, IL-1 $\beta$, IL-6, MRP14 (S100A9), CD11b, CD116, CD44, Galanin, CD54 (ICAM1), CCR7, GPR56, CD141, CD86, CD91 (LRP1), CD95, CD172a, Glut1, Glut5, TIM3, TIM4, Arginase-1 (Supplementary Table 5). Multiplexed and stained samples were simultaneously acquired on a CyTOF instrument. To validate the robustness of the results, we performed three independent measurements with a total of 36 huMG samples (summarized in Supplementary Table 1).

We used the commercially available analysis platform Cytobank (www.cytobank.org) to capture and visualize all huMG cells in a single two-dimensional (2D) map using unsupervised high-dimensional data analysis, the $\mathrm{t}$-distributed stochastic linear embedding ( $t-\mathrm{SNE}$ ) algorithm (Fig. 1b). In line with our previous evidence for regional heterogeneity of human microglia ${ }^{39}$, we observed a unique phenotype of SVZ microglia compared to microglia isolated from other brain regions (Fig. 1b, c). The huMG from control SVZ showed significantly higher expression of HLA-DR, CD11c, and CX3CR1 (Fig. 1c). Most importantly, we did not observe separation of huMG from donors with MDD and controls (Fig. 1b), which is in strong contrast to what we had previously observed for glioma-associated huMG ${ }^{45}$. No differences in the expression of HLA-DR, CD206, CD11c, CX3CR1, TNF, and CCL2 were observed for huMG from different brain regions between control and MDD cases (Fig. 1c). However, huMG from MDD cases expressed attenuated levels of HLA-DR in SVZ (Fig. 1c).

\section{Increased $\mathrm{P}_{2} \mathrm{Y}_{12}$ and TMEM119 expression in microglia from MDD brains}

Next, to fully harness the high-dimensionality of the mass cytometry data and to study huMG in more detail, we performed a comprehensive analysis on $\mathrm{R} /$ Bioconductor using the over-clustering approach ${ }^{40}$. Of note, the number of defined clusters may not solely represent biological functional subsets of huMG, but should be considered as an exploratory tool for the discovery of differential abundance of small/rare huMG populations between the two analyzed groups. All markers of antibody Panels $A$ and $B$ were included in the meta-clustering analysis, revealing eight distinct phenotype clusters of huMG (Fig. 2a-d). The defined clusters displayed a differential distribution across the different brain regions (Fig. 2c, d). The main cluster 2 (C2) was significantly less abundant in SVZ, whereas cluster 3 (C3) was more abundant in SVZ (Fig. 2d). No significant differences in cluster distribution were observed between huMG from MDD and control cases (Fig. 2d). 
a

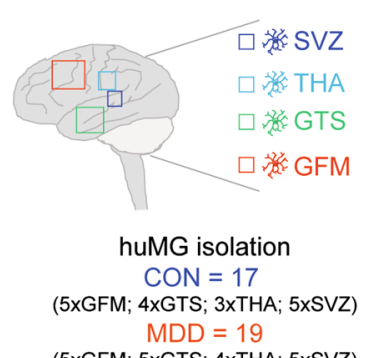

(5xGFM; 5xGTS; 4xTHA; 5xSVZ)

b

overlaid (regions) (all samples)

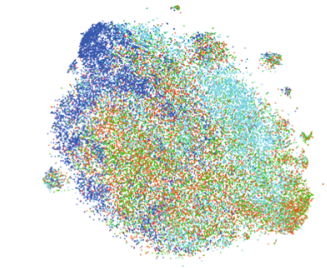

- SVZ • GTS $\bullet \mathrm{GFM} \bullet T H A$

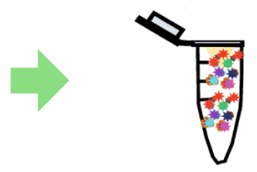

- barcoding

- sample pooling

- antibody staining - CyTOF measurement
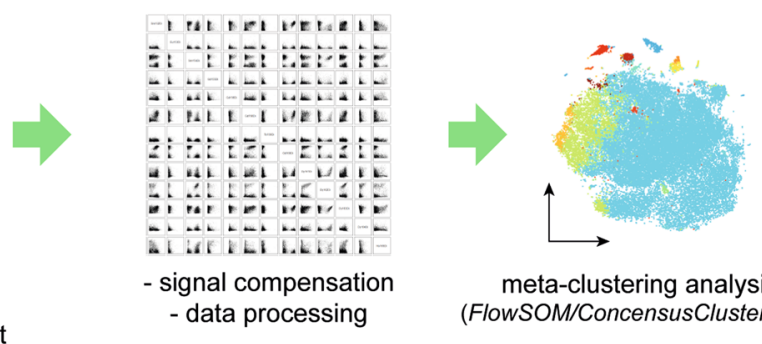

meta-clustering analysis (FlowSOM/ConcensusClusterPlus)

C
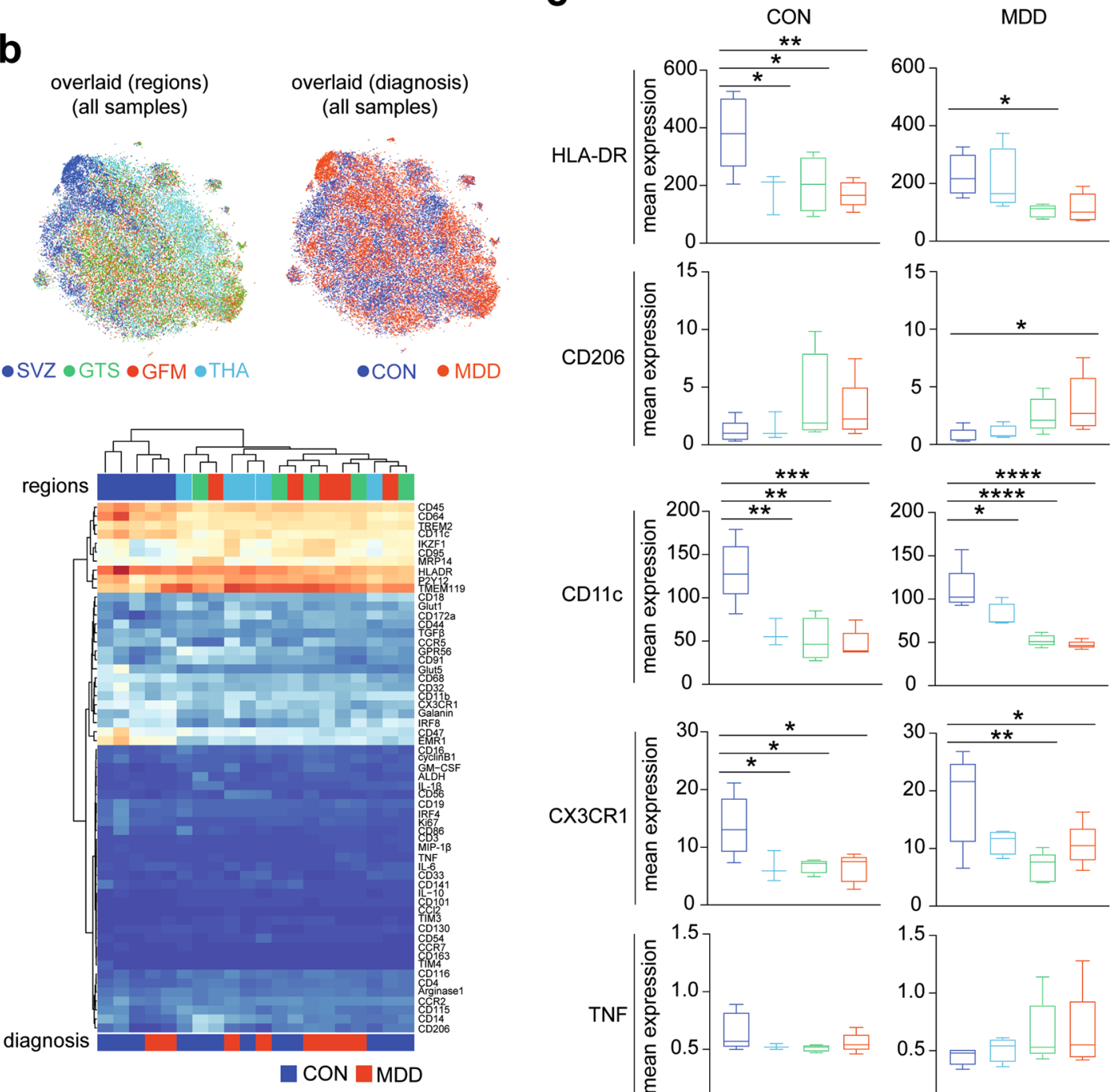
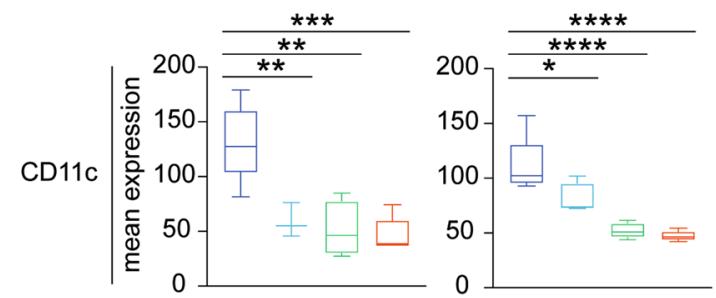

CX3CR1 $\mid$
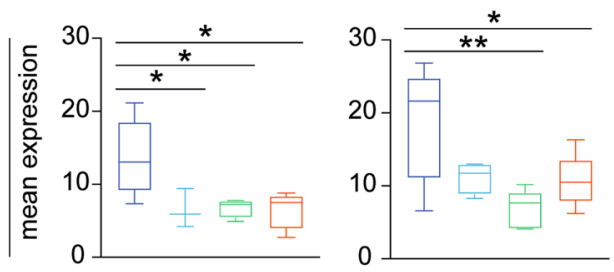

TNF
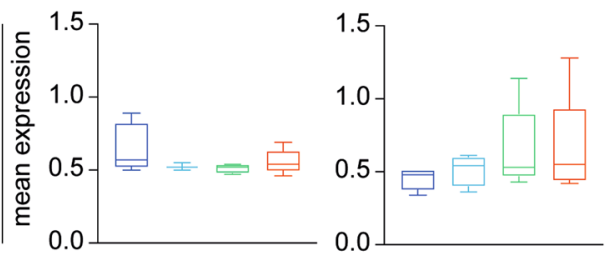

CCL2

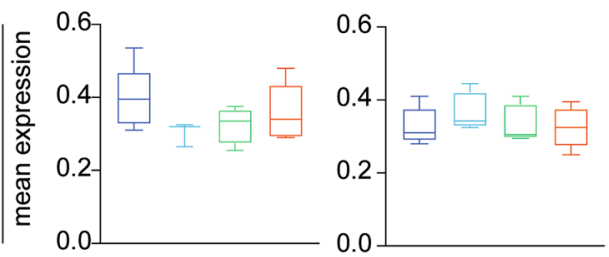

Fig. 1 (See legend on next page.) 
(see figure on previous page)

Fig. 1 Regional heterogeneity of microglia. a Schematic representation of the experimental workflow for CyTOF. Human microglia (huMG) were isolated from the subventricular zone (SVZ, $n=10)$, thalamus (THA, $n=7)$, temporal lobe $(\mathrm{GTS}, n=9)$, and frontal lobe $(\mathrm{GFM}, n=10)$ of eleven independent donors (CON, $n=5 ; \mathrm{MDD}, n=6$ ). HuMG samples were barcoded and pooled. Three different measurements of three different pooled samples were performed. Each pooled sample was divided in half and stained with two panels of metal-conjugated antibodies (Panels $A$ and $B$, Supplementary Tables 4 and 5) and measured on the CyTOF instrument. Prior to algorithm-based data analysis, the data were demultiplexed and compensated. Clustering analysis was performed to discover small phenotypic differences between the studied groups using algorithm-based data analysis workflow, FlowSOM/ConsensusClusterPlus. $\mathbf{b}$ The overlaid t-SNE plot of 16 samples (SVZ $=4$; THA $=4$, GTS $=4$; GFM $=4$ from two biologically independent CON donors and two biologically independent MDD donors) is shown. The coloring denotes different regions (left image) and studied groups (right image). The 2D t-SNE maps were generated based on expression levels of all markers of Panel A (Supplementary Table 4). The heat map cluster (bottom) demonstrates the expression levels of 59 analyzed markers. Samples are indicated by dendrograms, regions and diagnosis are colorcoded as above. Heat colors show overall expression levels (dark blue: no expression; red: high expression). c) Boxplots show mean expression levels of selected markers in different brain regions for huMG from CON and MDD cases. Boxes extend from the 25th to 75th percentiles. Whisker plots show the min (smallest) and max (largest) values. The line in the box denotes the median. ${ }^{*} P<0.05,{ }^{* *} P<0.01$, ${ }^{* * *} P<0.001$, ${ }^{* * *} P<0.0001$, one-way ANOVA testing with Dunnett correction for multiple comparisons. All brain regions were compared with the SVZ.

Comparison of microglia phenotypes within defined clusters revealed significant differences between MDD and control cases (Fig. 2e-g). Notably, the main cluster C2 contained huMG with significantly higher expression of the homeostatic markers $\mathrm{P}_{2} \mathrm{Y}_{12}$ and transmembrane protein (TMEM) $119^{46}$ in MDD compared to controls (Fig. 2e). At the same time, huMG from cluster C2 expressed lower levels of the activation markers HLA-DR and $\mathrm{CD} 68^{47}$ in MDD (Fig. 2e). HuMG from the SVZenriched cluster $\mathrm{C} 3$ also expressed more TMEM119 and less HLA-DR and CD68 in MDD cases (Fig. 2f). In contrast, CD206 ${ }^{\text {hi }}\left(\mathrm{P}^{2} \mathrm{Y}_{12}{ }^{\text {lo }}{ }^{\text {TMEM119 }}{ }^{\text {lo }}\right)$ macrophages from cluster C8 expressed more HLA-DR, TMEM119 and CD91 (LRP1) in MDD than control cases (Fig. 2g). Importantly, huMG/macrophages from clusters C2, C3, and $\mathrm{C} 8$ expressed higher levels of the chemokine CD195 (CLL5), but comparable levels of IL-1 $\beta$, IL-6, TNF, MIP$1 \beta$ (CCL4), IL-10, and MCP-1 (CCL2) between MDD and control cases (Fig. 2e-g). None of the other examined markers from Panels $A$ and $B$, including among others CX3CR1, IRF8, IRF7, CD11b, CD11c, CD86, CD45, TREM2, APOE, AXL, CD33 (Siglec-3), CD54 (ICAM1), CD56 (NCAM), GPR56, CD74, TGF $\beta$, IFN- $\alpha$, Galanin, CD95 (Fas), PD-L1, and Glut5, were differentially expressed between huMG/macrophages from MDD and control cases (data not shown).

\section{Discussion}

This is the first study to examine human microglia in MDD at the single-cell level. We found a noninflammatory phenotype of microglia with enhanced homeostatic functions in medicated MDD cases.

Our results are in line with recent microarray-based transcriptomic profiling of cerebral cortex in 87 cases of MDD, revealing no significant changes (FDR-corrected $P$ value $>0.05)$ in the gene expression of $H L A-D R, T N F$, IL6, IL1B, IL1A, IL3, IL5, IL8, IL10, ITGAM (CD11b), FKBP5, TLR2, CCL2, and CD14 compared with matched controls $^{48}$. Notably, the study detected increased transcript levels of $I L 17$ and CCL5, the latter binding to the chemokine receptor CCR5, which we found increased by CyTOF in human microglia from MDD brains. Interestingly, systemic inflammation can trigger CCR5dependent migration of microglia to the cerebral vasculature $^{49}$. Gene expression profiling of post-mortem frontal lobe tissue from 14 MDD cases showed increased expression of IL1A,IL3,IL5, IL8,IL10, but not IL6 or $T N F^{33}$. Gene expression of TNF, IL1B and $I L 10$ was also unchanged in the prefrontal cortex of depressed suicides $^{28}$. We have performed PCR analysis of myeloid cells from post-mortem brain tissue of 20 MDD cases compared with 27 controls and found no differential expression of $I L 6, I L 1 B$, and TNF mRNAs, even after in vitro challenge with lipopolysaccharide and dexamethasone (Snijders et al., submitted for publication). However, we detected increased expression of CX3CR1 and TMEM119 mRNAs, and decreased expression of CD163 and CD14 protein (Snijders et al., submitted for publication), underscoring the results of this single-cell CyTOF analysis of human microglia in the MDD brain.

The power of high-dimensional single-cell analysis lies in the precise identification of cell populations and the detection of rare disease-associated microglia states that would go undetected with bulk analysis and the use of single markers ${ }^{45,50-52}$. Even though the sample size is very small and medication effects cannot be excluded, our study suggests that homeostatic functions may be enhanced in MDD microglia. It is tempting to speculate that the increased expression of TMEM119 and P2Y 12 in microglia clusters from MDD cases may reflect enhanced neuron-microglia communication via transforming growth factor (TGF) $\beta 1$, which shows significant geneenvironment interactions predicting adult depression in the context of early life trauma ${ }^{53}$, as well as purines, in an attempt to protect neuronal function ${ }^{54,55}$. Along these lines, monocyte-derived microglia-like cells from individuals with schizophrenia exhibit increased synapse engulfment ${ }^{56}$. Expression of $\mathrm{P}^{2} \mathrm{Y}_{12}$ in microglia is 

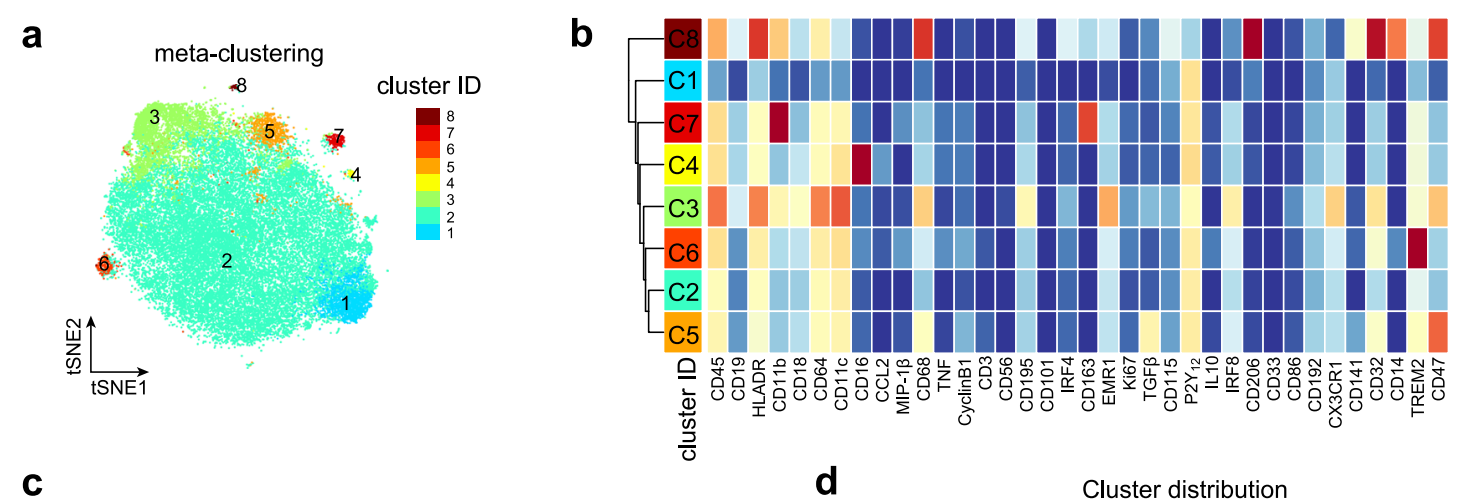

C

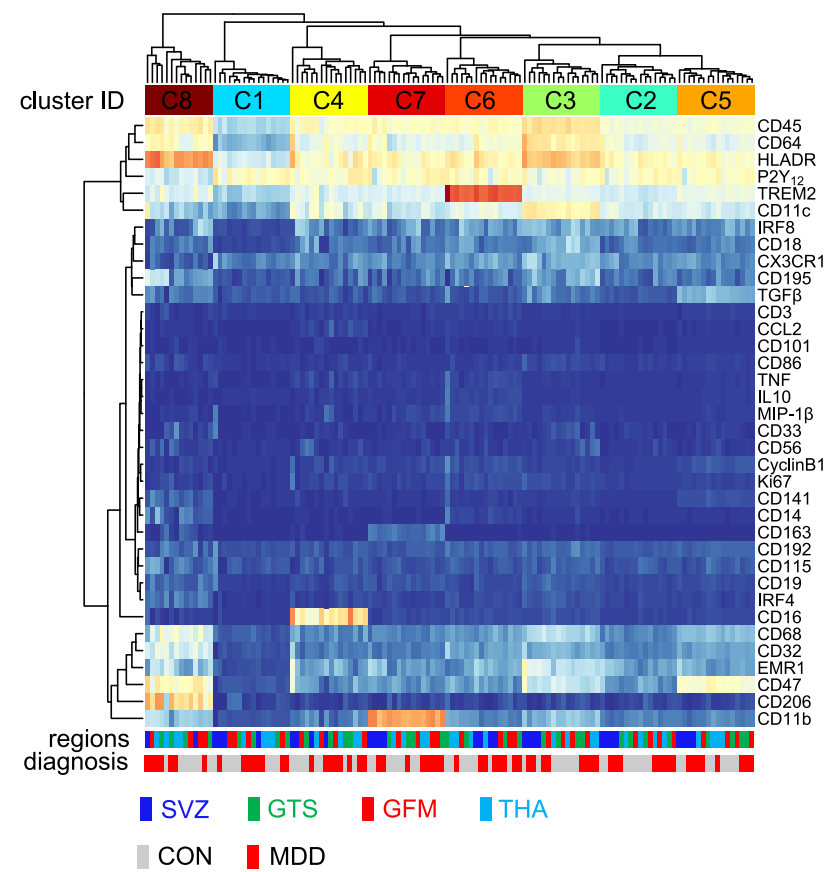

d

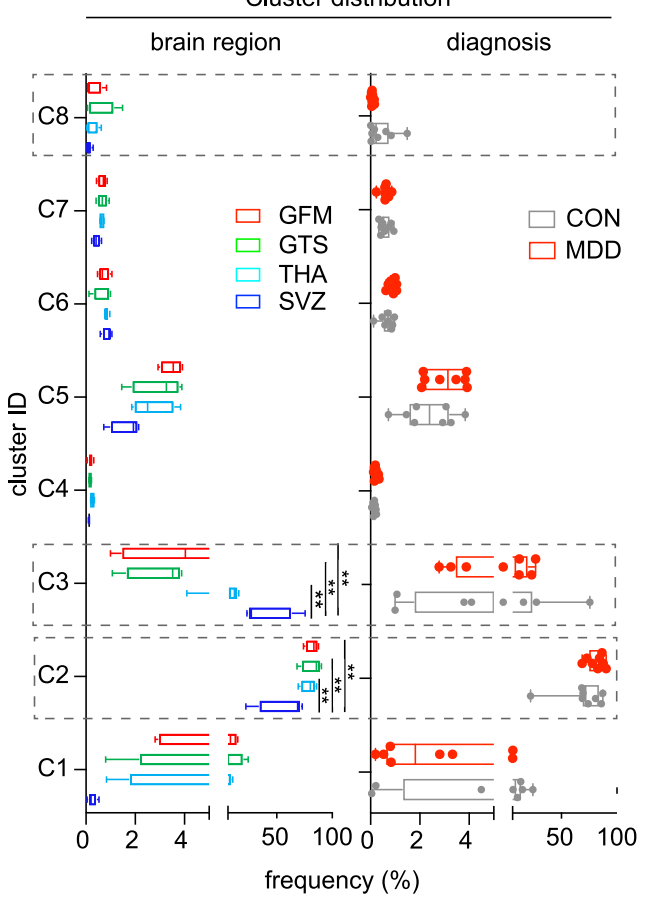

e main microglia cluster (C2)

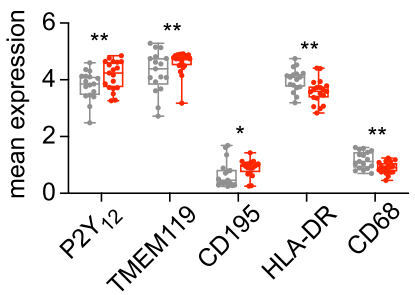

h

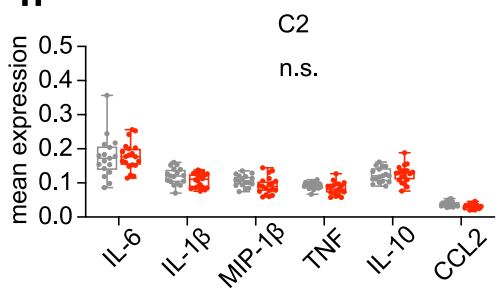

f

SVZ-abundant cluster (C3)
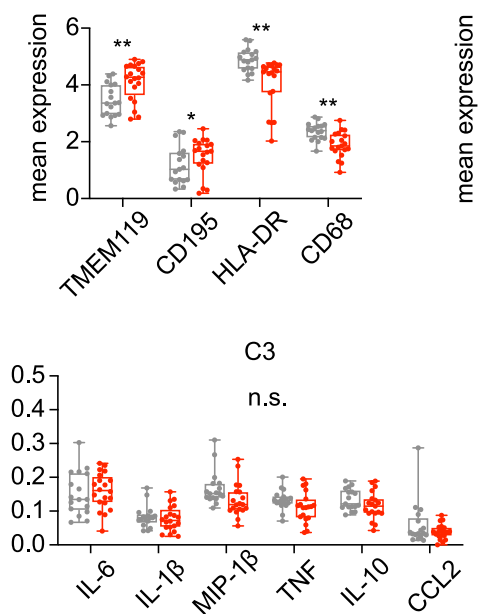

g $\mathrm{P} 2 \mathrm{Y}_{12} \mathrm{lo} /-$ (or TMEM119lo/-)

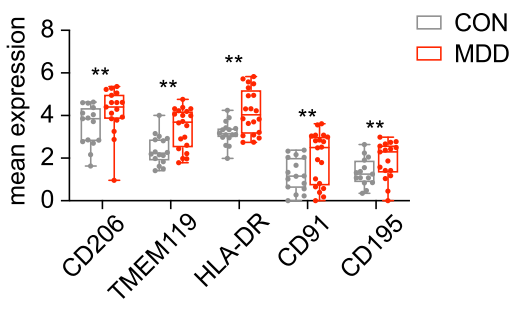

Fig. 2 (See legend on next page.) 
(see figure on previous page)

Fig. 2 Phenotypic diversity of myeloid cells in the MDD brain. a The overlaid t-SNE plot of 16 huMG samples (SVZ $=4 ; T H A=4 ; G T S=4 ; G F M=$ 4) isolated from four biologically independent donors $(C O N=2 ; M D D=2)$ is shown. The $2 D$ t-SNE maps were generated based on expression levels of all markers of Panel A (Supplementary Table 4). The coloring indicates eight defined clusters representing diverse myeloid cell phenotypes. $\mathbf{b}$ Heat map and cluster analysis of all samples demonstrates the phenotypes of all eight defined clusters on the basis of the mean expression levels of 36 markers used for the cluster analysis. Identified clusters are indicated by dendrograms. Heat colors show overall marker expression levels (red: high expression; dark blue: no expression). c Heat map cluster demonstrates the expression levels of 36 analyzed markers for all eight clusters of each sample. Samples are indicated by dendrograms. Heat colors show overall expression levels (dark blue: no expression; red: high expression). d Boxplots on the left show the cluster distribution across four different brain regions (SVZ, blue; THA, light blue; GTS, green; GFM, red) and on the right the cluster distribution for the two groups (CON and MDD). Whisker plots show the min (smallest) and max (largest) values. A dot indicates the frequency (\%) of an individual sample. The line in the box denotes the median. ${ }^{* *} P<0.01$, multiple t-test with FDR adjustment (at $10 \%$ using the Benjamini-Krieger-Yekutieli procedure). (e-g) Boxplots showing markers with differential expression (arcsinh) between CON and MDD for (e) the main microglia cluster $\mathrm{C} 2,(\mathbf{f})$ the SVZ-enriched huMG cluster C3, and (g) the P2Y $12^{\mathrm{lo} /-}$ (or TMEM119 ${ }^{\mathrm{lo} /-}$ ) macrophage cluster C8. A dot indicates the mean expression of an individual sample from all three measurements. ${ }^{*} P<0.05,{ }^{*} P<0.01$, multiple t-test with FDR adjustment (at $10 \%$ using the Benjamini-Krieger-Yekutieli procedure). $\mathbf{h}$ Boxplots showing selected cytokines and chemokines (IL-6, IL-1 $\beta$, MIP-1 $\beta$, TNF, IL-10, CCL2) that are not differentially expressed (arcsinh) between CON and MDD for the clusters C2, C3, and C8. A dot indicates the mean expression of an individual sample from all three measurements. A $P$ value $<0.05$ was considered statistically significant (multiple t-test with FDR adjustment at $10 \%$ using the Benjamini-Krieger-Yekutieli procedure).

important for synaptic plasticity ${ }^{57}$ and for adult hippocampal neurogenesis ${ }^{58}$, which has been associated with the response to antidepressant treatment ${ }^{59}$. Neuronal activity regulates the surveilling function of microglia processes in the cortex via the monoamine neurotransmitter norepinephrine $e^{60,61}$, which is reduced in the brains of patients with depression ${ }^{62,63}$. A reduction in norepinephrine tone results in microglial extension and territory surveillance ${ }^{60}$. Interestingly, microglia were found to be hyper-ramified in a chronic despair mouse model of depression, and this morphological change was mediated by neuron-microglia signaling via CX3CR1, and restored by antidepressant treatment ${ }^{64}$.

Neuroinflammation is associated with the marked downregulation of homeostatic markers like $\mathrm{P}_{2} \mathrm{Y}_{12}$ and TMEM119 in microglia ${ }^{52,65,66}$. In contrast, we found that both markers were increased in microglia from MDD brains and none of the proinflammatory mediators were altered, calling into question the presence of an active inflammatory process. In fact, we even detected a downregulation of the immune molecules, HLA-DR and CD68, in the main cluster of microglia from MDD brains compared with controls. HLA-DR is a major histocompatibility complex (MHC) class II molecule involved in antigen presentation, which is constitutively expressed by human microglia with higher expression levels in white than gray matter ${ }^{45,67}$. Expression of HLA-DR is strongly induced in activated microglia across a variety of neuroinflammatory and neurodegenerative diseases ${ }^{52,68}$, and axonal damage in multiple sclerosis has been associated with HLA-DR ${ }^{+}$microglia ${ }^{69}$. CD68 is a glycoprotein that primarily localizes to the endosomal/lysosomal compartment, but also acts as a class D scavenger receptor on the plasma membrane of monocytes/macrophages ${ }^{70}$. Human microglia constitutively express CD68 with higher expression levels in white than gray matter ${ }^{39,45}$. Microglial expression of CD68 is strongly induced by neuroinflammation ${ }^{52,71}$, which may also represent a tipping point in the pathogenesis of Alzheimer's disease ${ }^{72}$. Interestingly, the brains of individuals resilient to dementia despite robust Alzheimer's neuropathology (amyloid plaques and neurofibrillary tangles) displayed lower numbers of $\mathrm{CD} 68^{+}$microglia in the temporal lobe and higher levels of the cytokines IL- 6 , IL- $1 \beta$, IL- $10^{73}$. Recent evidence from genome-wide association studies suggests shared genetic architecture between MDD and late-onset Alzheimer's disease $^{74,75}$, and some of the identified genes were highly expressed in monocytes/macrophages and involved in immune response and endocytosis. However, the findings of our high-dimensional single-cell analysis of microglia in MDD do not lend support to neuroinflammatory changes in any of the examined cortical and subcortical brain regions. The results are in line with earlier studies of candidate markers like HLA-DR and CD68 in postmortem brain tissue from MDD cases with and without suicidality $^{28-32}$.

Our study has several limitations. The sample size is very small due to the difficulties in obtaining sufficient quality post-mortem brain tissue for CyTOF analysis. We were particularly interested in comparatively assessing different brain regions as human microglia are diverse, and we were able to replicate our earlier findings of regional heterogeneity of human microglia ${ }^{39}$ in this independent cohort. Although microglia from different post-mortem brain regions did not differ between MDD and controls, the attenuated expression of HLA-DR in microglia from the microenvironment of the subventricular zone may warrant further exploration. An important study has recently found that the brain transcriptional profile of MDD differs greatly by gender; men with MDD exhibited increases in oligodendrocyte- and microglia-related genes, while women with MDD had 
decreases in these markers ${ }^{76}$. Notably, $P 2 R Y 12$ expression in the anterior cingulate cortex was increased in men with MDD and decreased in women with MDD compared to controls $^{76}$. Our sample size was too small to correct for gender effects, but we did not observe different response patterns of microglia (including expression of $\mathrm{P} \mathrm{Y}_{12}$ ) between the three women and three men with MDD versus controls in our sample. Another confounder of our study may be the effects of medication, in particular antidepressants, on immune responses in the brain. It is well known that tricyclic antidepressants, selective serotonin reuptake inhibitors (SSRI), and serotonin and noradrenaline reuptake inhibitors (SNRI) can reduce the expression of proinflammatory cytokines like IL-6, IL-1 $\beta$, and TNF- $\alpha$, and increase the expression of IL-10, but the opposite effects have also been described ${ }^{77}$. Moreover, many of these studies examined peripheral blood and the cytokine changes may actually reflect treatment response. We cannot control for the effects of the diverse range of medication in our small sample, but it is important to note that the majority of MDD cases (at least 4/6) were clinically depressed at the time of death based on retrospective chart analysis and one case did not receive antidepressant medication during the last 3 months before death. Furthermore, $24 \mathrm{~h}$ before death, both control and MDD donors received morphine, opioids, sedatives and/or anesthetic agents (Supplementary Table 2). It has been demonstrated that these drugs may exert suppressive effects on the immune system and impair monocyte/macrophage function ${ }^{78-80}$. However, as a common limitation of studies performed in humans, the effects reported in the literature were variable and potentially confounded by different methods used to assess immune responses, large spectrum of drugs with different dosages, and low numbers of study participants $^{80}$. In the future, it will be important to comparatively assess the effects of medication on peripheral immune cells and microglia in MDD by controlled trials. Finally, we were also unable to control for agony, comorbid conditions, and bias introduced by diagnosis based on retrospective medical chart review by two independent psychiatrists.

The results of this first high-dimensional single-cell analysis of microglia in MDD provide a missing piece in the concept of neuroinflammation in mood disorders. Further validation in larger cohorts and the use of additional techniques like single-cell RNA sequencing are required to ascertain the findings. Our results may be of particular value for PET studies which have relied on TSPO ligand binding to determine microglial responses in the CNS. Our findings also raise the intriguing possibility that supporting the functions of microglia in the brain may be more beneficial in MDD than the use of antiinflammatory agents.

\section{Acknowledgements}

The authors thank the Psychiatric Donor Program of the Netherlands Brain Bank (NBB-Psy) for providing human tissue samples. This work was supported by grants from the German Research Foundation (SFB/TRR167 B05 to C.B., B05 and B07 to J.P.; SFB/TRR265 B04 to J.P.) and the UK Dementia Research Institute (Momentum Award to J.P.). Open Access funding enabled and organized by Projekt DEAL.

\begin{abstract}
Author details
'Department of Neuropsychiatry and Laboratory of Molecular Psychiatry, Charité - Universitätsmedizin Berlin, Berlin, Germany. ${ }^{2}$ Department of Psychiatry, Brain Center Rudolf Magnus, University Medical Center Utrecht, Utrecht, The Netherlands. ${ }^{3} \mathrm{BIH}$ Center for Regenerative Therapies (BCRT), Charité - Universitätsmedizin Berlin, Berlin, Germany. ${ }^{4}$ Department of Psychiatry, Icahn School of Medicine at Mount Sinai, New York, NY, USA. ${ }^{5}$ Flow \& Mass Cytometry Core Facility, Charité - Universitätsmedizin Berlin and Berlin Institute of Health (BIH), 10178 Berlin, Germany. ${ }^{6}$ Mental IIIness Research, Education and Clinical Center (MIRECC), James J Peters VA Medical Center, Bronx, NY, USA. ${ }^{7}$ DZNE and BIH, Berlin, Germany. ${ }^{8}$ University of Edinburgh and UK Dementia Research Institute (DRI), Edinburgh, UK
\end{abstract}

Conflict of interest

The authors declare that they have no conflict of interest.

\section{Publisher's note}

Springer Nature remains neutral with regard to jurisdictional claims in published maps and institutional affiliations.

Supplementary Information accompanies this paper at (https://doi.org/ 10.1038/s41398-020-00992-2).

Received: 17 April 2020 Revised: 10 August 2020 Accepted: 21 August 2020 Published online: 11 September 2020

\section{References}

1. GBD 2017 Disease and Injury Incidence and Prevalence Collaborators. Global, regional, and national incidence, prevalence, and years lived with disability for 354 diseases and injuries for 195 countries and territories, 1990-2017: a systematic analysis for the Global Burden of Disease Study 2017. Lancet 392, 1789-1858 (2018).

2. Kupfer, D. J., Frank, E. \& Phillips, M. L. Major depressive disorder: new clinical, neurobiological, and treatment perspectives. Lancet 379, 1045-1055 (2012).

3. Wray, N. R. et al. Genome-wide association analyses identify 44 risk variants and refine the genetic architecture of major depression. Nat. Genet. 50, 668-681 (2018).

4. Chan, R. F. et al. Cell type-specific methylome-wide association studies implicate neurotrophin and innate immune signaling in major depressive disorder. Biol. Psychiatry 87, 431-442 (2020).

5. Peyrot, W. J. et al. Does childhood trauma moderate polygenic risk for depression? A meta-analysis of 5765 subjects from the Psychiatric Genomics Consortium. Biol. Psychiatry 84, 138-147 (2018).

6. Normann, C. \& Buttenschøn, H. N. Gene-environment interactions between HPA-axis genes and stressful life events in depression: a systematic review. Acta Neuropsychiatr. 31, 186-192 (2019).

7. Zannas, A. S. et al. Epigenetic upregulation of FKBP5 by aging and stress contributes to NF-KB-driven inflammation and cardiovascular risk. Proc. Natl Acad. Sci. USA 116, 11370-11379 (2019).

8. Wohleb, E. S., Franklin, T., Iwata, M. \& Duman, R. S. Integrating neuroimmune systems in the neurobiology of depression. Nat. Rev. Neurosci. 17, 497-511 (2016).

9. Leonard, B. E. Inflammation and depression: a causal or coincidental link to the pathophysiology? Acta Neuropsychiatr. 30, 1-16 (2018).

10. Enache, D., Pariante, C. M. \& Mondelli, V. Markers of central inflammation in major depressive disorder: A systematic review and meta-analysis of studies examining cerebrospinal fluid, positron emission tomography and postmortem brain tissue. Brain Behav. Immun. 81, 24-40 (2019).

11. Miller, A. H., Maletic, V. \& Raison, C. L. Inflammation and its discontents: the role of cytokines in the pathophysiology of major depression. Biol. Psychiatry 65 , 732-741 (2009). 
12. Culmsee, $C$. et al. Mitochondria, microglia, and the immune system-how are they linked in affective disorders? Front. Psychiatry 9, 739 (2019).

13. Himmerich, H., Patsalos, O., Lichtblau, N., Ibrahim, M. A. A. \& Dalton, B. Cytokine research in depression: principles, challenges, and open questions. Front. Psychiatry 10, 30 (2019).

14. Clark, S. L. et al. A methylation study of long-term depression risk. Mol. Psychiatry https://doi.org/10.1038/s41380-019-0516-z (2019).

15. Euteneuer, F. et al. Peripheral immune alterations in major depression: the role of subtypes and pathogenetic characteristics. Front. Psychiatry $\mathbf{8}$, 250 (2017).

16. Köhler, C. A. et al. Peripheral alterations in cytokine and chemokine levels after antidepressant drug treatment for major depressive disorder: systematic review and meta-analysis. Mol. Neurobiol. 55, 4195-4206 (2018).

17. Arteaga-Henríquez, G. et al. Low-grade inflammation as a predictor of antidepressant and anti-inflammatory therapy response in MDD patients: a systematic review of the literature in combination with an analysis of experimental data collected in the EU-MOODINFLAME Consortium. Front. Psychiatry 10, 458 (2019)

18. Syed, S. A. et al. Defective inflammatory pathways in never-treated depressed patients are associated with poor treatment response. Neuron 99, 914-924.e3 (2018).

19. Drexhage, R. C. et al. Inflammatory gene expression in monocytes of patients with schizophrenia: overlap and difference with bipolar disorder. A study in naturalistically treated patients. Int. J. Neuropsychopharmacol. 13, 1369-1381 (2010).

20. Carvalho, L. A. et al. Inflammatory activation is associated with a reduced glucocorticoid receptor alpha/beta expression ratio in monocytes of inpatients with melancholic major depressive disorder. Transl. Psychiatry 4, e344 (2014).

21. Grosse, L. et al. Clinical characteristics of inflammation-associated depression: Monocyte gene expression is age-related in major depressive disorder. Brain Behav. Immun. 44, 48-56 (2015).

22. Leday, G. G. R. et al. Replicable and coupled changes in innate and adaptive immune gene expression in two case-control studies of blood microarrays in major depressive disorder. Biol. Psychiatry 83, 70-80 (2018).

23. Zhang, $H$. X. et al. Difference in proinflammatory cytokines produced by monocytes between patients with major depressive disorder and healthy controls. J. Affect. Disord. 234, 305-310 (2018).

24. Alvarez-Mon, M. A. et al. Abnormal distribution and function of circulating monocytes and enhanced bacterial translocation in major depressive disorder. Front. Psychiatry 10, 812 (2019).

25. Prinz, M., Jung, S. \& Priller, J. Microglia Biology: one century of evolving concepts. Cell 179, 292-311 (2019).

26. Clark, S. M. et al. Dissociation between sickness behavior and emotionality during lipopolysaccharide challenge in lymphocyte deficient Rag2(-/-) mice. Behav. Brain Res. 278, 74-82 (2015).

27. Steiner, J. et al. Severe depression is associated with increased microglial quinolinic acid in sub regions of the anterior cingulate gyrus: evidence for an immune-modulated glutamatergic neurotransmission? J. Neuroinflamm. 8, 94 (2011).

28. Torres-Platas, S. G., Cruceanu, C., Chen, G. G., Turecki, G. \& Mechawar, N. Evidence for increased microglial priming and macrophage recruitment in the dorsal anterior cingulate white matter of depressed suicides. Brain Behav. Immun. 42, 50-59 (2014).

29. Bayer, T. A., Buslei, R., Havas, L. \& Falkai, P. Evidence for activation of microglia in patients with psychiatric illnesses. Neurosci. Lett. 271, 126-128 (1999).

30. Hamidi, M., Drevets, W. C. \& Price, J. L. Glial reduction in amygdala in major depressive disorder is due to oligodendrocytes. Biol. Psychiatry 55, 563-569 (2004).

31. Steiner, J. et al. Immunological aspects in the neurobiology of suicide: elevated microglial density in schizophrenia and depression is associated with suicide. J. Psychiatr. Res. 42, 151-157 (2008).

32. Brisch, R. et al. Microglia in the dorsal raphe nucleus plays a potential role in both suicide facilitation and prevention in affective disorders. Eur. Arch. Psychiatry Clin. Neurosci. 267, 403-415 (2017).

33. Shelton, R. C. et al. Altered expression of genes involved in inflammation and apoptosis in frontal cortex in major depression. Mol. Psychiatry 16, 751-762 (2011).

34. Setiawan, E. et al. Role of translocator protein density, a marker of neuroinflammation, in the brain during major depressive episodes. JAMA Psychiatry 72, 268-275 (2015).
35. Li, H., Sagar, A. P. \& Kéri, S. Microglial markers in the frontal cortex are related to cognitive dysfunctions in major depressive disorder. J. Affect. Disord. 241, 305-310 (2018).

36. Setiawan, E. et al. Association of translocator protein total distribution volume with duration of untreated major depressive disorder: a cross-sectional study. Lancet Psychiatry 5, 339-347 (2018).

37. Owen, D. R. et al. Pro-inflammatory activation of primary microglia and macrophages increases $18 \mathrm{kDa}$ translocator protein expression in rodents but not humans. J. Cereb. Blood Flow. Metab. 37, 2679-2690 (2017)

38. Perry, V. H. Microglia and major depression: not yet a clear picture. Lancet Psychiatry 5, 292-294 (2018)

39. Böttcher, $\mathrm{C}$. et al. Human microglia regional heterogeneity and phenotypes determined by multiplexed single-cell mass cytometry. Nat. Neurosci. 22, 78-90 (2019).

40. Böttcher, C. et al. Multi-parameter immune profiling of peripheral blood mononuclear cells by multiplexed single-cell mass cytometry in patients with early multiple sclerosis. Sci. Rep. 9, 19471 (2019).

41. Chevrier, $\mathrm{S}$. et al. Compensation of signal spillover in suspension and imaging mass cytometry. Cell Syst. 6, 612 (2018).

42. Nowicka, M. et al. CyTOF workflow: differential discovery in high-throughput high-dimensional cytometry datasets. Version 2. F1000Res. 6, 748 (2017).

43. Van Gassen, S. et al. FlowSOM: Using self-organizing maps for visualization and interpretation of cytometry data. Cytom. A 87, 636 (2015).

44. Wilkerson, M. D. \& Hayes, D. N. ConsensusClusterPlus: a class discovery tool with confidence assessments and item tracking. Bioinformatics 26, 1572-1573 (2010).

45. Sankowski, R. et al. Mapping microglia states in the human brain through the integration of high-dimensional techniques. Nat. Neurosci. 22, 2098-2110 (2019).

46. Butovsky, O. et al. Identification of a unique TGF- $\beta$-dependent molecular and functional signature in microglia. Nat. Neurosci. 17, 131-143 (2014).

47. Hendrickx, D. A. E., van Eden, C. G., Schuurman, K. G., Hamann, J. \& Huitinga, I. Staining of HLA-DR, Iba1 and CD68 in human microglia reveals partially overlapping expression depending on cellular morphology and pathology. J. Neuroimmunol. 309, 12-22 (2017).

48. Gandal, M. J. et al. Transcriptome-wide isoform-level dysregulation in ASD, schizophrenia, and bipolar disorder. Science 362, eaat8127 (2018).

49. Haruwaka, K. et al. Dual microglia effects on blood brain barrier permeability induced by systemic inflammation. Nat. Commun. 10, 5816 (2019).

50. Keren-Shaul, $H$. et al. A unique microglia type associated with restricting development of alzheimer's disease. Cell 169, 1276-1290 (2017).

51. Krasemann, S. et al. The TREM2-APOE pathway drives the transcriptional phenotype of dysfunctional microglia in neurodegenerative diseases. Immunity 47, 566-581 (2017).

52. Masuda, T. et al. Spatial and temporal heterogeneity of mouse and human microglia at single-cell resolution. Nature 566, 388-392 (2019).

53. Cattaneo, $\mathrm{A}$. et al. FoxO1, A2M, and TGF- $\beta 1$ : three novel genes predicting depression in gene $X$ environment interactions are identified using crossspecies and cross-tissues transcriptomic and miRNomic analyses. Mol. Psychiatry 23, 2192-2208 (2018).

54. Attaai, A. et al. Postnatal maturation of microglia is associated with alternative activation and activated TGF $\beta$ signaling. Glia 66, 1695-1708 (2018).

55. Cserép, C. et al. Microglia monitor and protect neuronal function through specialized somatic purinergic junctions. Science 367, 528-537 (2020).

56. Sellgren, C. M. et al. Increased synapse elimination by microglia in schizophrenia patient-derived models of synaptic pruning. Nat. Neurosci. 22, 374-385 (2019).

57. Sipe, G. O. et al. Microglial P2Y12 is necessary for synaptic plasticity in mouse visual cortex. Nat. Commun. 7, 10905 (2016).

58. Diaz-Aparicio, I. et al. Microglia actively remodel adult hippocampal neurogenesis through the phagocytosis secretome. J. Neurosci. 40, 1453-1482 (2020).

59. Park, S. C. Neurogenesis and antidepressant action. Cell Tissue Res. 377, 95-106 (2019).

60. Liu, Y. U. et al. Neuronal network activity controls microglial process surveillance in awake mice via norepinephrine signaling. Nat. Neurosci. 22, 1771-1781 (2019).

61. Stowell, R. D. et al. Noradrenergic signaling in the wakeful state inhibits microglial surveillance and synaptic plasticity in the mouse visual cortex. Nat Neurosci. 22, 1782-1792 (2019). 
62. Post, R. M., Gordon, E. K., Goodwin, F. K. \& Bunney, W. E. Jr. Central norepinephrine metabolism in affective illness: MHPG in the cerebrospinal fluid. Science 179, 1002-1003 (1973).

63. Lambert, G., Johansson, M., Agren, H. \& Friberg, P. Reduced brain norepinephrine and dopamine release in treatment-refractory depressive illness: evidence in support of the catecholamine hypothesis of mood disorders. Arch. Gen. Psychiatry 57, 787-793 (2000).

64. Hellwig, S. et al. Altered microglia morphology and higher resilience to stressinduced depression-like behavior in CX3CR1-deficient mice. Brain Behav. Immun. 55, 126-137 (2016).

65. Mildner, A., Huang, H., Radke, J., Stenzel, W. \& Priller, J. P2Y12 receptor is expressed on human microglia under physiological conditions throughout development and is sensitive to neuroinflammatory diseases. Glia $\mathbf{6 5}, \mathbf{3 7 5 - 3 8 7}$ (2017).

66. Zrzavy, T. et al. Loss of 'homeostatic' microglia and patterns of their activation in active multiple sclerosis. Brain 140, 1900-1913 (2017).

67. Gehrmann, J., Banati, R. B. \& Kreutzberg, G. W. Microglia in the immune surveillance of the brain: human microglia constitutively express HLA-DR molecules. J. Neuroimmunol. 48, 189-198 (1993).

68. McGeer, P. L., Itagaki, S. \& McGeer, E. G. Expression of the histocompatibility glycoprotein HLA-DR in neurological disease. Acta Neuropathol. 76, 550-557 (1988).

69. Huizinga, R. et al. Phagocytosis of neuronal debris by microglia is associated with neuronal damage in multiple sclerosis. Glia 60, 422-431 (2012).

70. Chistiakov, D. A., Killingsworth, M. C., Myasoedova, V. A., Orekhov, A. N. \& Bobryshev, Y. V. CD68/macrosialin: not just a histochemical marker. Lab Invest 97, 4-13 (2017)
71. Wierzba-Bobrowicz, T. et al. Morphological analysis of active microglia-rod and ramified microglia in human brains affected by some neurological diseases (SSPE, Alzheimer's disease and Wilson's disease). Folia Neuropathol. 40, 125-131 (2002)

72. Streit, W. J. et al. Microglial activation occurs late during preclinical Alzheimer's disease. Glia 66, 2550-2562 (2018)

73. Barroeta-Espar, I. et al. Distinct cytokine profiles in human brains resilient to Alzheimer's pathology. Neurobiol. Dis. 121, 327-337 (2019).

74. $\mathrm{Ni}, \mathrm{H}$. et al. The GWAS risk genes for depression may be actively involved in Alzheimer's Disease. J. Alzheimers Dis. 64, 1149-1161 (2018).

75. Lutz, M. W., Sprague, D., Barrera, J. \& Chiba-Falek, O. Shared genetic etiology underlying Alzheimer's disease and major depressive disorder. Transl. Psychiatry 10, 88 (2020).

76. Seney, M. L. et al. Opposite molecular signatures of depression in men and women. Biol. Psychiatry 84, 18-27 (2018).

77. Kopschina Feltes, P. et al. Anti-inflammatory treatment for major depressive disorder: implications for patients with an elevated immune profile and nonresponders to standard antidepressant therapy. J. Psychopharmacol. 31, 1149-1165 (2017).

78. Prud'homme, G. J., Glinka, Y. \& Wang, Q. Immunological GABAergic interactions and therapeutic applications in autoimmune diseases. Autoimmun. Rev 14, 1048-1056 (2015).

79. Sedghi, S., Kutscher, H. L., Davidson, B. A. \& Knight, P. R. Volatile anesthetics and immunity. Immunol. Invest. 46, 793-804 (2017).

80. Franchi, S., Moschetti, G., Amodeo, G. \& Sacerdote, P. Do all opioid drugs share the same immunomodulatory properties? A review from animal and human studies. Front. Immunol. 10, 2914 (2019). 